\title{
Philosophiques
}

\section{Le temps de la nature et la mesure du temps}

\section{Dominique Janicaud}

Volume 23, numéro 2, automne 1996

URI : https://id.erudit.org/iderudit/027400ar

DOI : https://doi.org/10.7202/027400ar

Aller au sommaire du numéro

Éditeur(s)

Société de philosophie du Québec

ISSN

0316-2923 (imprimé)

1492-1391 (numérique)

Découvrir la revue

Citer cet article

Janicaud, D. (1996). Le temps de la nature et la mesure du temps Philosophiques, 23(2), 327-339. https://doi.org/10.7202/027400ar d'utilisation que vous pouvez consulter en ligne.

https://apropos.erudit.org/fr/usagers/politique-dutilisation/ 


\title{
INTERVENTIONS
}

\section{LE IEMPS DE LA NATURE Et LA MESURE DU IEMPS}

\author{
PAR \\ DOMINI QUE JANICAUD
}

La question du temps constitue certainement l'un des problèmes philosophiques les plus difficiles. Cette difficulté se révèle aussi bien au niveau phénoménal (comment saisir la phénoménalité temporelle, alors que - comme Kant l'a remarqué - le temps "n'est pas un concept empirique qui dérive d'une expérience quelconque " ?) qu'au niveau proprement spéculatif (comment définir le temps et comment penser les paradoxes qu'il engendre?).

D'un autre côté, ce nœud de questions présente l'avantage de ne pas se réduire à un problème philosophique trop "technique "sans aucun rapport avec l'expérience quotidienne. En fait, c'est un problème à "double entrée ": nous pouvons l'aborder par une réflexion sophistiquée, mais aussi par des observations phénoménologiques presque accessibles à tout un chacun.

Dans le cadre restreint du présent propos, je repartirai de ce niveau aisément abordable, afin de trouver un angle d'attaque concernant la question du temps physique et de sa mesure. Dois-je préciser que, mème en me limitant à cet aspect de la question, je ne prétends pas offrir ici une vision synoptique de la problématique du temps physique, mais seulement ouvrir une perspective de réflexion à partir de quelques remarques touchant à la mesure du temps ?

Ricoeur déplore, dans Temps et récit, que la phénoménologie contemporaine ait dissocié le temps cosmologique du temps de la conscience. Il a raison. Et l'on peut mème aller plus loin : un temps phénoménologique est-il concevable sans aucune référence au temps naturel ? Inversement, le temps naturel est-il livré tout fait par les choses, sans intentionnalité humaine? Le temps physique n'est-il qu'un ensemble de mesures exterieures dont on pourrait se passer ou, au contraire, constitue-t-il la base même de toute perception et conscience du temps?

Repartons d'abord des éléments naturels pour déterminer s'ils nous offrent, à défaut du temps lui-mème, des indices, des traces, des virtualités annonçant ou rendant possible la mesure du temps. C'est en inversant cette démarche, c'est-à-dire en repartant des techniques de mesure du temps (et de leur histoire) que se révélera la production du temps physique par sa mesure, le côté fabriqué du 
temps physique. Finalement, de quoi prendra-t-on mesure, si cette mesure même est temporalisation? Ne s'agira-t-il pas de l'événement?

\section{Quel temps de la nature?}

Parler d'un temps de la nature, c'est avoir en vue un temps qui échappe aux aléas de l'introspection et des différences d'appréciation subjective, un temps censé palpiter au cœur de la nature elle-même, en sa présence immédiate aussi bien qu'en son ampleur cosmique. La nature a-t-elle une unité, mais aussi une âme et un cœur, comme l'a cru le romantisme allemand ? Une telle représentation résiste-t-elle à l'examen? En fait, ni notre évolution individuelle depuis l'enfance, ni l'expérience commune, même repensée par la phénoménologie, n'offre une temporalité physique unifiée.

Les éléments naturels perdurent. Mais qu'entendre par là ? Fautil prèter le même temps à la terre, au ciel, à l'eau, au feu ? La réponse nous paraît aujourd'hui aller de soi, mais cette évidence nous fait oublier toutes les étapes qu'il a fallu franchir pour atteindre le niveau d'une chronométrie unifiée. Nous oublions aussi facilement le fait que les éléments naturels nous ont précédés immémorialement et nous survivront incommensurablement.

À supposer qu'il fasse sens, le concept de "temps de la nature " implique à la fois les préalables d'une abstraction et d'une capacité de mesure, double elaboration consciente conquise sur l'horizon qui cerne notre existence. Sans invalider le concept de "temps physique ", elle permet d'en souligner le caractère paradoxal : s'il y a un temps de la nature, il est justement ce que l'immédiateté naturelle dérobe. Le temps de la nature en soi s'avère inconnaissable. Aucun des cinq sens ne nous livre le temps comme tel : nous voyons, entendons, humons, touchons, goûtons dans le temps, mais non le temps. Nous ne sentons que les effets du temps. Il nous faut d'abord en quelque sorte épeler le temps en faisant l'épreuve de sa négativité à l'égard du monde sensible, en découvrant son invisibilité ou son "inscrutabilité " (comme le dit Ricoeur).

Dès lors qu'appelons-nous, à tort ou à raison, "temps physique "? Tentons maintenant d'interroger l'expérience existentielle, sans présupposer le concept d'une nature unifiée, en repartant simplement des quatre éléments : terre, ciel, eau, feu.

Le spéléologue qui s'enfonce au cœur de la terre perd le sens de notre temps pour découvrir une temporalité "longue". Les expériences de Michel Siffre ont permis de mieux connaitre les rythmes physiologiques, en particulier celui du sommeil (le rythme dit bicircadien : 36 heures d'éveil et 12 heures de sommeil). Ces plongées "hors temps" ont révélé que le seul repère temporel qui subsiste alors est notre corps fonctionnant comme une "horloge interne ".

Les trois autres éléments offrent ou proposent des repères apparemment plus nets. Encore faut-il les observer, les isoler, les interpréter. C'est le cas du ciel qu'il ne suffit pas d'admirer ou de contempler béatement, de l'eau qui en s'écoulant sans ètre canalisée ou réglée n'offre aucune marque, enfin du feu qui est l'instabilité même et dont l'incandescence risque de ne laisser aucune trace exploitable, seulement des cendres pulvérisées... 
Les premiers enseignements de cette enquète encore rudimentaire sont négatifs : aucun de nos sens ni aucun élément naturel ne livre par lui-mème cette dimension étrange et spécifique que nous nommons " temps ". Celle-ci n'émerge que grâce à des indices, des traces, des effets dont l'homme prend progressivement le contröle.

Voyons maintenant, en reprenant chacun des éléments, comment ces occasions offertes par la nature trouvent forme, grāce à la mesure.

Les horloges telluriques sont parmi les plus anciennes. L'écoulement réglé du sable, le glissement lent d'un poids permettent d'utiliser la pesanteur pour jauger des irréversibilités simples.

Avant mème l'invention des clepsydres perfectionnées qui émerveillèrent les Anciens, des vases creux, de volume connu, se remplissant graduellement, avaient permis d'utiliser l'eau pour mesurer le temps.

Le feu, en soi si indomptable, permet de lire le temps, s'il brūle un bâtonnet d'encens, une chandelle graduée ou s'il habite une lampe à huile dont la réserve est connue.

Le ciel, enfin, ouvre sur le cosmos. Son observation est bien plus ancienne que notre histoire. Les lunaisons et la position des astres offrent des repères cycliques plus complets et moins fragiles que les indices telluriques'.

La condition essentielle de la conjonction entre naturalité et temporalité se rêvèle bien dans la mesure. Point de conscience temporelle sans cette mise en relation; et point de mesure sans le secours de repères naturels. La temporalisation résulte de ce chassécroisé.

Il serait cependant hâtif de ne pas discerner, sous le terme général de "mesure", des degrés très diversifiès du sens chronologique. Les peuples "sans histoire "peuvent avoir une perception très fine des durées, sans disposer d'une èchelle temporelle générale et homogène. Ainsi, au $X^{e}{ }^{e}$ siècle, fait prisonnier par les Indiens Tuppinambas, un marin allemand note: "Pour fixer l'époque du depart, ils disent : c'est quand telle espèce de fruit sera mûre ; car ils n'ont aucune autre manière de désigner les années et les jours. Ils choisissent ordinairement, pour leur départ, l'époque du frai d'une espèce de poisson qu'ils appellent pratti...2."

Entre ces appréciations surtout qualitatives du temps et notre obsession de la précision, une longue histoire s'est déroulée. Il a fallu du temps pour mesurer le temps. Quels enseignements tirer de cette histoire?

\section{Quelques leçons de l'histoire de la mesure du temps}

Mue par un souci apparemment louable d'"authenticité ", la philosophie contemporaine du temps n'a eu que trop tendance à mépriser le "temps des horloges". Il en résulta une schématisation excessive, voire caricaturale, de la quotidienneté temporelle et un

1. Voir sur ces aspects du temps naturel notre conférence donnée à Lausanne en aoūt 1994 (à paraître dans les Actes de ce Congrès) : "Le temps de la nature et la nature du temps". Nous venons d'en reprendre quelques élëments.

2. Voir Hans Staden, Nus, féroces et anthropophages, Paris, Seuil-Points, 1990. p. 205. 
rejet du temps "lui-même " ou de la durée "pure "dans l'abstraction. Lui restituer son importance est donc nécessaire et légitime, à condition de ne pas se contenter d'une mise au point qui se réduirait elle-même à une simple formalité. Puisque le sens étroit (quantitatif) et le sens large (l'appréciation) communiquent, on ne saurait faire une histoire de la mesure du temps en se contentant de considérations purement techniques. L'inventivité en ce domaine a été favorisée et mème rendue possible par des changements radicaux dans les attitudes et dans l'attention mème à la durée. Comme le remarque David Landes : "Ce n'est pas l'horloge qui a provoqué un intérêt pour la mesure du temps; c'est l'intérêt pour la mesure du temps qui a conduit à l'invention de l'horloge $\mathrm{e}^{3}$ ". A rebours, les perfectionnements de la chronométrie ont puissamment contribué à bouleverser les expériences existentielles de la temporalité et continuent à le faire. Sous ces deux perspectives, l'amont et l'aval, notre méditation a beaucoup à gagner d'une réflexion sur l'histoire de la mesure du temps.

En amont du "temps des horloges ", la situation est loin d'ètre simple, aussi bien du point de vue historique qu'au regard des enseignements de l'anthropologie. Tout d'abord, il n'est pas inutile de préciser que nous entendons désigner par l'expression consacrée, "temps des horloges" (et, semble-t-il, avec nous des philosophes comme Bergson et Heidegger) le temps continu, divisible en unités homogènes, offrant des repères quantitatifs précis pour l'appréciation de phénomènes successifs. Computé par des montres ou horloges mécaniques à échappement, ce temps ne connait un degré de précision comparable au nôtre que depuis le XVIII siècle; il aura fallu quatre siècles pour perfectionner l'horloge mécanique qui, "à ses débuts, fut un instrument grossier, imprécis et peu fiable " Mais, objectera-t-on, on a eu l'idée de ce temps homogène bien avant que la technique pût le mettre à la disposition de tout un chacun sous la forme de machines sūres et précises. Le concéder ne fait que déplacer le problème : remontons au XIV siècle ou même plus avant au cœur du Moyen-Äge ; portons aussi notre regard sur des peuples non-occcidentaux. Que constatons-nous? Non une indifférence, une ignorance complètes et immémoriales vis-à-vis de la temporalité, faisant passer d'un seul coup d'une durée qualitative au temps quantitatif tel que nous le connaissons. "Il n'importait pas, en Chine, de connaitre l'heure avec précision " : cette remarque de David Landes peut ètre étendue à une myriade de civilisations et de peuplades chez lesquelles l'absence de la notion de temps homogène n'allait nullement de pair avec le manque de tout sens de la durée ni même avec l'indifférence à toute maîtrise de celle-ci. Au contraire, pourrait-on soutenir: les expériences de la durée devaient se diversifier considérablement ; d'autre part, la fabrication fort ancienne de garde-temps rudimentaires révèle un intérèt pour une mesure, au moins minimale, du temps.

En mettant l'accent sur l'importance de la nostalgie des origines et la mise au point de nombreuses techniques de retour en arrière (qui

3. David S. Landes, L'heure qu'il est, op. cit., p. 98.

4. Ibid., p. 133.

5. Ibid., p. 79. 
vont des initiations ou thérapeutiques visant une "nouvelle naissance "aux mystiques indo-chinoises du "retour en arrière "), Mircea Eliade n'a pas voulu dire que l'homme archaique ignorait les effets d'usure du temps ni toute différenciation de ses rythmes effectifs ${ }^{6}$ : la loi du karma indien est celle du temps pensé comme source de destruction et de souffrance. Eliade confirme ce que nous apprend d'un autre côté l'histoire des mesures du temps : les temps mythiques, les rituels liés aux grands cycles saisonniers ou cosmiques révèlent un désir, encore plus profond que toute demande de mesure chronologique, de maitriser la durée multiforme en ses méandres et en ses inconnues. Si l'inconscient est intemporel ${ }^{7}$, le regressus ad uterum s'affirme comme une aspiration enracinée au coeur de nos pulsions, inéliminable et d'une puissance inouie ${ }^{8}$. Le plus surprenant n'est donc pas que l'humanité ait - si longtemps et sous toutes les latitudes - ignorè notre temps homogène ; ce qui est difficile à comprendre, et capital de préciser, c'est l'inverse : qu'une idée aussi peu naturelle (et affectivement peu gratifiante) que celle de ce temps homogène ait pu finalement s'imposer avec la puissance irrésistible que nous lui connaissons. Comment de nouvelles demandes ont-elles peu à peu appelè, facilité, exigé, puis imposé cette forme de maitrise chronométrique (à double tranchant) à partir d'unités homogènes de plus en plus fines?

L'Europe n'a pas été la seule à vouloir reproduire et prédire le temps astral ni mème à fabriquer des machines compliquées pour mesurer le temps (l'horloge de Su Song, fabriquée en Chine au $X^{e}$ siècle, en tèmoigne) ${ }^{9}$. Elle a été la seule à dégager un intérêt pour la fonction temporelle comme telle, en son abstraction, et à concentrer ses efforts pour faire converger cet intérèt avec un perfectionnement patient des instruments de mesure du temps ${ }^{10}$. De cette convergence résulteront évidemment de considérables effets en retour, quand les horloges, puis les montres se généraliseront.

Mais, avant d'en arriver là, comment une demande "chronologique "s'est-elle exercée assez intensément pour faire sortir le monde médiéval de sa torpeur rurale ? Landes insiste sur le rôle du temps monastique bénédictin et surtout cistercien. L'existence mème d'une " règle "implique une organisation imposant des obligations fixes, et non plus seulement articulées au gré des tāches agricoles, dans ces monastères qui sont les entreprises productives de l'époque. Ce facteur économique, en un sens assez large, est luimême conditionné et renforcé par une conception métaphysique : le but unique, le salut de l'âme, impose une sorte de rentabilité du temps : la brièveté de la vie humaine ne saurait être gaspillée ; toute heure compte pour le rachat des péchés. La bonne organisation des

6. Voir Mircea Eliade, Aspects du mythe, Paris, Gallimard, 1963 et en particulier le chap. V : "Le temps peut être maitrisé *.

7. D'après Freud. Voir Marie Bonaparte, Chronos. Eros. Thanatos, Paris, PUF, 1952, p. 24.

8. "Pour se guérir de l'œuvre du temps, il faut "revenir en arrière " et rejoindre le "commencement du monde" ": c'est ainsi qu'Eliade caractérise cette tendance qui lui parait pénétrer et traverser toutes les cultures et tous les àges (op. cit., p. 110).

9. D. S. Landes, op. cit., p. 51 ss.

10. Ibid., p. 95. 
offices n'est donc pas la seule raison d'un souci de la mesure régulière du temps. Invoquer, d'ailleurs, la pression de la mêtaphysique chrétienne est encore trop général : c'est un certain christianisme, cistercien et ascétique, qui accorde valeur au travail productif. Le christianisme à lui seul ne saurait expliquer ces annonces de la modernité technicienne : le christianisme des Béatitudes, le christianisme augustinien ou franciscain sont indifferents ou peu attentifs au temps du travail, mais bien plutōt à ses annonces eschatologiques. Au demeurant, il serait étrange que l'on ait dû attendre plus de dix siècles pour que le christianisme révèle son souci de la "productivité "!

Ce qui est certain, c'est qu'on voit apparaitre dès le Moyen-Āge une discipline temporelle liee à l'organisation d'un travail collectif et productif. Le seuil suivant, franchi beaucoup plus près de nous, aux $\mathrm{XVII}^{e}$ et $\mathrm{XVIIl}^{\mathrm{e}}$ siècles ${ }^{11}$, correspond à une demande d'un type apparemment tout autre (la recherche de la précision dans la navigation hauturière), mais qui répond, elle aussi, à un souci d'accumulation de richesses et de moyens monétaires. La monstration du temps (qui le phénoménalise) est toujours intéressée ; mais ici, cet intérèt se focalise sur une accumulation de type nettement capitaliste : le contrôle du temps devient un facteur direct de rentabilité dans des proportions qui transcendent désormais le travail de petits groupes humains. Le temps computé avec précision se détache de l'homme pour acquérir une valeur en soi, support des autres valeurs économiques, et devenir le ressort décisif de la course à la puissance.

Au moins deux leçons se dégagent de ce trop rapide coup d'œil sur l'histoire de la mesure du temps. En premier lieu, si notre "temps des horloges" est simple dans ses principes (la continuité, l'homogénéité et l'irréversibilité - ce dernier trait correspondant à l'insertion du quantitatif pur dans la dimension chronologique et calendaire -, sa mise au point technique a supposé une extraordinaire somme d'inventions de détail et de perfectionnements durant des siècles. Pour atteindre le degré de sūreté et de précision que nous connaissons, il a fallu dompter le plus rétif et le plus indocile des phênomènes.

En second lieu, à un niveau plus proprement philosophique, il devient impossible de soutenir, au vu de cette histoire, - d'une part que le "temps des horloges "forme une sorte de bloc monolithique, d'autre part que l'évaluation chronologique (au sens large) ne dépend pas en très grande partie des changements intervenus dans la mesure effective du temps (au sens étroit). À l'affirmation bergsonienne selon laquelle "le temps qui dure n'est pas mesurable ${ }^{12}$ ", nous devons opposer le fait que la mesure du temps a commandé sa phénoménalisation. Cette thèse implique que, sans nier les décrochements considérables entre les expériences existentielles du temps (encore aujourd'hui, tout le monde n'obéit pas, Dieu merci, à la temporalité la plus " abstraite ", par exemple celle d'un spéculateur

11. Voir Landes, op. cit., p. 168-176. Une pléiade de savants illustres a concentré ses efforts, au XVII ${ }^{e}$ siècle, sur le problème des longitudes ( $c f$. ibid., p. 175).

12. Bergson, Durée et simultanéité in Mélanges, op. cit. p. 102. 
boursier ou d'un pilote de jet intercontinental), il n'y ait pas de durée dont l'intériorité échapperait, comme par miracle, à ce mouvement de détermination chronologique qui change les rythmes vitaux et influence jusqu'aux sensibilités. Il n'y a pas de temps caché derrière ses actes de naissance et de croissance (en l'occurrence, des déterminations de plus en plus précipitées). Les temporalisations sont bien celles qui sont rendues possibles dans le champ technicopratique. Les prises de conscience de la temporalité et leur intensification ne sont pas séparables des supports qui les expriment. Cependant, toute pression implique une résistance : dans le cas présent, une résistance humaine, car l'intérêt pratique individuel ne converge pas forcément, selon une intensité uniforme, avec le principe de rentabilité qui gouverne l'économie du temps dans le monde occidental développé.

La limite de l'histoire est notre présent mouvant. Cette frontière est elle-mème temporelle. Les leçons de l'histoire de la mesure du temps doivent ètre complétées par l'examen du front sans cesse déplacé de cette "guerre du temps"que l'homme moderne semble condamné à mener toujours plus intensément.

\section{L'apprentissage de l'incommensurabilité}

Il serait tentant de soutenir que les modifications quantitatives, mẽme si elles correspondent à de fantastiques degrés de précision, ne changent rien au fait qu'elles restent quantitatives : elles ne font que reculer une limite qui n'est nullement celle du temps lui-même (en son essence), mais seulement de sa mesure. Notre effort a été de résister à cette tentation et ce qui précède nous a fourni des arguments à cet effet. L'inconvénient majeur du mépris envers le temps quantitatif est de dissocier le temps cosmologique du temps phénoménologique. Or ceux-ci n'ont pas seulement un "droit égal ", comme le soutient Ricoeur ${ }^{13}$ : ils représentent les deux pôles du procès de temporalisation, qui n'est jamais purement ni naturelcosmologique, ni intentionnel-phénoménologique.

Il n'est pas indifférent que la conquête de la précision chronologique transforme considérablement l'économie de notre temporalité vécue: la discipline temporelle, de plus en plus intériorisée, contamine jusqu'à nos activités de loisir. Ce qui est encore plus décisif, c'est la portée imaginaire-symbolique de ces transformations qui aboutissent au retoumement paradoxal du mesurable en incommensurable. L'homme se heurte à ce que Junger a nommé le "mur du temps" et il en est conscient. Ce "mur " est perceptible d'abord dans la position même de la question d'un "temps élémentaire": si, en-dessous de $10^{34}$, toutes les relations (y compris la gravitation) deviennent indifférenciables, n'y a-t-il pas - au-delà mème de l'ordre de la picoseconde atteint en chimie - une limite quantique extrême de la "précision "temporelle autour de $10^{-24} ?^{14}$ Plus généralement, si nous jetons un coup d'œil sur les résultats obtenus sur l'ensemble impressionnant des mesures qui vont de l'infiniment petit à l'infiniment grand, un constat doit

13. Paul Ricoeur, Temps et récit, op. cit., 3, p. 164.

14. Voir Manfred Eigen, "Evolution und Zeitlichkeit ", art. cit., p. 39. 
donner à penser : l'extraordinaire extension des échelles de mesure confronte, presque à chaque pas, avec l'incommensurable et, du mème coup, oblige à repenser la portée de ces mesures.

Peut-on avoir la même vue sur l'homme quand on le croit créé "il y a plus de quatre mille ans ", ou issu d'une évolution de millions d'années ? La même question se pose vis-à-vis du cosmos, cette foisci à l'échelle de milliards d'années. À ce propos, l'usage trop répandu de l'expression " histoire de l'univers", pour abusif qu'il soit (puisqu'il n'y a, au sens strict, d'histoire qu'humaine), devrait cependant rappeler que le temps cosmique est toujours mesuré par des hommes et surtout que sa signification imaginaire-symbolique hante nos existences et déborde constamment l'énonciation de pures " données".

L'explosion chronologique qui a modifié l'horizon scientifique depuis Darwin, concernant les origines de l'homme, n'est pas réductible à un simple changement d'échelle. Ou plutôt, celui-ci est tel que nous "n'en sommes pas encore revenus ": nous n'avons pas encore pris mesure de l'incommensurable dont les bouleversements des mesures étaient porteurs. Rendus possibles par des progrès techniques (stratigraphie, radioactivité, radioastronomie, etc.), ces résultats doivent être interprétés et pensés. Mème si l'homme est toujours à cet égard au-dessous de ses tâches, méme si la science laissée à elle-mème a tendance à ne pas penser ses propres résultats, de nouveaux investissements symboliques pénètrent notre imaginaire : c'est la "maison univers " qui s'ouvre et s'élargit au-delà du concevable. L'extension fabuleuse des échelles temporelles n'est pas séparable de la diversification de leurs significations ${ }^{15}$.

Affrontant cette incommensurabilité du temps, Jean Cocteau, toujours fureteur, attribue cet échange à Langevin et Einstein : " mais comment mesurez-vous ces choses? "demande le premier; "ces choses ne se mesurent pas " répond le second; et Cocteau de suggérer que le temps est "un phénomène de perspective analogue à celui de la tète de mort de Holbein ${ }^{16}$ ". La profonde justesse de cette intuition concerne la finitude de notre expérience temporelle let même la finité du concept de temps rapporté à la réversibilité des lois physiques fondamentales) : une seconde peut sembler une éternité, à l'instar d'un atome recélant un univers de particules; sous une perspective radicalement élargie, une vie humaine a une durée négligeable en comparaison des trois millions d'années de l'existence de l'homme sur cette terre, durée à son tour infime au regard de l'āge de l'univers. Ces variations de perspective peuvent ètre multipliées à l'envi : l'immensité nous guette virtuellement en chacun de nos pas, en chacune de nos perceptions. Manfred Eigen s'est plu à montrer que les chiffres qui expriment l'àge de l'univers et ceux de la durée d'une vie humaine sont proches et les subdivisions de leurs "épisodes "comparables $\left(2.10^{10}\right.$ dans le premier cas ; $3.10^{9}$ dans le deuxième), à condition de préciser que les unités de compte sont respectivement l'année et la seconde. Cette relativité de toute appréciation temporelle ne doit cependant pas conduire à la neutralisation de l'expérience temporelle elle-mēme. Si toute prise de

15. Voir P. Ricoeur, op. cit., p. 167.

16. Jean Cocteau, Joumal d'un inconnu, Paris, Grasset, 1953, p. 167, 176. 
distance est instructive, et mēme irremplaçable, comment éviter qu'une vue de surplomb risque de brouiller la juste perspective recherchée?

Une fois signalé ce risque, reconnaissons que, trois siècles après les Pensées, il serait impardonnable de ne pas comprendre que l'infiniment grand et l'infiniment petit sont présents en l'homme, de manière encore bien plus fine et démultipliée que ne l'avait imaginé Pascal. L'incommensurabilité physique n'est sans doute encore qu'un signe d'une immensité encore plus surprenante : celle de la découverte d'un autre ordre, celui de l'événement. Pour Pascal, cet événement est l'incarnation de Jésus-Christ. Pour l'historien. l'événement n'est pas aussi singulier ou impondérable, mais il n'est pas aussi facile à cerner que le laisse supposer l'usage courant du mot. La frontière entre le mesurable et l'incommensurable est d'une extrème mobilité. Le plus incommensurable ne se glisse-t-il pas en nos actes et nos jugements sous la forme de l'événement?

\section{Quelle mesure pour l'èvénement?}

La question ne concerne pas uniquement l'historien fou le philosophe de l'histoire). Advenir, n'est-ce pas le geste temporel par excellence ? Une pierre n'advient pas; mais qu'une marque y soit traceee, cette pierre fait soudain signe pour des ètres temporels. En advenant, l'événement fait prendre forme à l'avenir immédiat : il fait surgir hic et nunc une forme unique et déterminée. Cependant, si l'événement temporalise avec une singulière intensité, la forme pure du temps n'est point par elle-même événement. Il y a, dans le devenir-événement, quelque chose qui dépend de nous, qui se fait signifiant pour nous et par nous.

Est événement tout ce "qui arrive ": définition beaucoup trop vague et qui peut s'appliquer aussi bien à une bataille décisive entre grands Etats qu'à un accident de la circulation, voire à un choc entre particules subatomiques. Cette généralité est-elle levée si l'on retient le caractère discriminant de la discontinuité, comme le fait Pomian lorsqu'il définit l'événement comme une "discontinuité révéla$\operatorname{trice}^{17}$,?

Discontinuitê : ce trait parait tout à fait pertinent, puisqu'il permet de surmonter le manque de rigueur de l'histoire événementielle qui s'en tenait au "simple narré des accidents ${ }^{18}$ ". L'histoire non critique, tout comme le mythe, est scandée de moments ou de ruptures dont le choix relève du bon vouloir de l'auteur, ou de la beauté du récit, plus que des ressorts du déploiement historique. De fait, l'événement n'est jamais livré par le temps lui-mème ; il est un produit de l'histoire et surtout, au premier chef, de l'historien, de sa subjectivité, voire de son arbitraire. En axant sa définition sur la "discontinuité ", Pomian ouvre l'événement sur des temporalités longues et rend justice à son invisibilité en l'insérant dans les structures où il intervient comme rupture, inflexion d'une conjoncture. Il préserve ainsi la dimension temporelle en la faisant échapper à la ponctualité de l'événement au sens traditionnel, par exemple une bataille, une visite d'ambassadeur

17. Krzysztof Pomian, L'ordre du temps, Paris, Gallimard, 1984, p. 30 ss.

18. Expression du Sieur de la Popelinière, citée par Pomian, ibidem p. 8. Voir supra, p. 68 ss, une première approche de la question de l'événement. 
ou un traité. Depuis Simiand et l'École des Annales, l'histoire contemporaine n'a-t-elle pas appris à déchiffrer, sous le flot tumultueux des changements apparents ou sensationnels, la stratification d'évolutions beaucoup plus lentes et profondes (modifications climatiques, circuits monétaires et économiques, réseaux techniques, permanence ou inflexion des institutions et des mentalités, etc.) ? Cependant, en postulant que l'événement désigne aussi bien une discontinuité au sein d'un modèle que l'irruption d'une nouveauté dans un horizon intersubjectif vécu, on ne fait que déplacer la difficulté qui concerne le caractère " révélateur " ou "significatif " de l'événement. Certes l'intérèt de l'élargissement de l'aire de l'événement est de surmonter les limites visibles, subjectives et arbitraires de l'événement pittoresque ou sensationnel : le changement de direction d'une courbe peut receler des conséquences bien plus décisives que la déchirure de l'événement ponctuel. Encore fautil que le point de rupture ou de "catastrophe" de la courbe soit compris, interprété, inséré dans un réseau d'informations et d'interrogations délimitant un horizon de problèmes. "Révélatrice ", la discontinuité ne l'est que pour un observateur apte à l'intégrer dans une trame sémantique. Là réside le noyau le plus énigmatique de l'événement, qu'il soit microscopique ou macroscopique, ponctuel ou immergé dans une "chronologie longue ".

Donner sens à un phénomène temporel, marquer l'instant, c'est créer un événement. Du moins est-ce vouloir le creer : cet essai devient trop aisément dérisoire, si l'intention ne rencontre aucun répondant. Qu'il s'agisse du camaval de Romans ou de l'évolution du commerce des blés, quel que soit le degré de discontinuité du phénomène envisagé, toujours se repose la question préalable lou devrait-elle se poser) : à partir de quel horizon de sens l'événementialité de l'événement se détache-t-elle et mérite-t-elle de retenir l'attention? C'est sous cet angle que mesure est prise d'un evénement.

Le danger trop évident, à ce point de la réflexion, est de voir se dissoudre l'événementialité de l'événement au moment même où l'on avait précisé ses conditions d'apparition: dissolution dans le foisonnement des possibles et dans l'arbitraire des subjectivités. Lhorizon du sens est sans limites, la temporalité une immense réserve d'événements à des niveaux multiples et à un degré inimaginable (ce qui permet de confirmer à quel point le sens du temps s'enracine dans notre chrono-fiction).

Un exemple simple permettra de mieux saisir la différence (et la frontière mouvante) entre mesure du temps et incommensurabilité de l'événement, afin de nous rapprocher du but que nous visons : préciser la relation entre sens et mesure dans le cas de l'événement. Ma montre m'offre un spectacle monotone et absolument prévisible : la ronde de la trotteuse, le parcours régulier et circulaire des aiguilles sur le cadran ; cette chronométrie exclut de son champ tout événement. Survienne une panne (due, par exemple, à l'usure de la pile de cette montre à quartz) : voici un tout petit événement, extêrieur à la chronométrie elle-même. "Discontinuité révélatrice " assurement, remarquera-t-on avec Pomian: la panne m'oblige à m'interroger et à opérer un renvoi au système technique au sein 
duquel s'insère ma montre. Si celle-ci était encore mécanique, je devrais me dire : "peut-être ai-je oublié de la remonter ? ".

Cet exemple n'invalide pas la définition que nous examinons ; il confirme ce que nous avancions sur la question du sens ; il a, en outre, l'avantage de préciser la relation entre mesure et incommensurabilité à partir de l'horizon chronométrique : dans ce cas précis, la panne n'est nullement "incommensurable "en soi ; elle oblige seulement à décrocher du niveau du fonctionnement chronométrique à celui du principe même de ce fonctionnement. À supposer qu'on veuille garder cette référence technicienne, un "événement " sera une rupture qui oblige à passer d'un niveau de fonctionnement donné à un niveau "supérieur", ou simplement différent, pour inventer une procédure ou une explication permettant d'obtenir un résultat (censé bloqué au premier niveau). Mais cette rupture doit ètre comprise et intégrée dans une procédure ou un nouveau système de renvois.

Gue nos exemples soient prélevés sur un système technique en général, ou sur un système chronométrique en particulier, ils incitent, de toute façon, à renverser la définition de l'événement comme "discontinuité révélatrice": celle-ci ne peut ètre identifiée comme discontinuité qu'à l'intérieur d'un système de renvois ou d'un projet qui sont les lieux possibles de la "révélation " en question. Nous n'avons pas d'abord affaire à une discontinuité, puis à une révélation; mais inversement : une anticipation de sens, un projet, une attente permettent l'identification d'une rupture et en font un événement, fût-il de modeste portée.

Avons-nous ainsi éclairé quelque peu la limite de l'incommensurabilité de l'événement ? Si nous revenons à l'exemple de la montre en prenant en compte le préalable du projet (c'est-à-dire de l'horizon temporel comme halo d'attentes), nous constatons que l'événement est biface : d'une part, il s'insère dans la régularité qu'il rompt (il sera donc relatif au temps de la montre); d'autre part, il prend sa dimension d'événement d'une ouverture sur l'incertain, ou plutōt sur l'écheveau des grilles d'intelligibilité (la panne est-elle ordinaire? ne révèle-t-elle pas un type de dysfonctionnement auquel je n'avais pas songé et pour lequel je ne suis pas compétent? quelqu'un a-t-il touché à la montre ? et pourquoi ? etc.).

L'événement n'est donc "discontinuité révélatrice "que pour autant qu'il s'insère dans un projet, c'est-à-dire au sein d'un horizon temporel (dans le cas d'un événement " raconté "par un historien, cet horizon est redoublé : l'horizon de l'historien suppose celui des acteurs de l'événement passé). Cette précision paraît inutile parce qu'évidente ; elle permet pourtant de faire échapper la "discontinuité révélatrice "à son excessive généralité. Toute inflexion d'une courbe ne fait pas événement, toute discontinuité non plus. Dans l'événement, le caractère "révélateur " est étroitement lié à la dimension temporelle (sans que celle-ci suffise à créer l'événement, puisque tout instant ne fait pas événement).

Notre effort de spécification peut s'appuyer sur un exemple célèbre repris par Kojève (César franchissant le Rubicon) pour qualifier l'événement historique. César se promène au bord de la petite rivière : il n'y a là rien d'historique. La promenade ne revêt ce caractère que si elle s'insère dans un projet dont la portée concerne 
le destin de Rome : "L'événement en question ne serait donc pas “ historique " s'il n'y avait pas une présence-réelle (Gegenwart) de l'avenir dans le Monde réel (tout d'abord dans le cerveau de César) ${ }^{19}$ ". Il faut être encore plus radical que Kojève, si l'on ne considère pas uniquement l'événement au sens historico-mondial, mais tout événement qui se détache sur l'indiffèrenciation des instants : par exemple, un jeune homme se promène au bord du Rubicon pour méditer sur un chagrin d'amour. C'est un "non événement * du point de vue historique ; c'est pourtant un événement pour sa famille et ses intimes. Préalablement à la dimension historique proprement dite et la rendant possible, il faut donc faire place à la dimension de l'historicité, c'est-à-dire du temps faisant sens.

L'historien objectera qu'il a affaire à des événements précis, à des faits - qu'ils relèvent d'une temporalité longue ou ponctuelle -, mais que la notion d'historicité ne "lui sert à rien ". Nous répondrons qu'il en est de mème déjà du temps qui, dans sa généralité, est présupposé par l'historien. En fait, la discipline historique a deux présuppositions (dont elle n'a cure tant qu'elle opère sur des positivités) : le temps et l'historicité. Elle les fait intervenir et les explicite uniquement lorsqu'elle en a besoin au sein de ses champs d'investigation narratifs-explicatifs.

Il subsiste cependant une difficulté proprement philosophique qui tient à la relation que nous avons postulée entre temporalité et mesure : rẻintroduire l'historicité comme condition de l'événement, n'est-ce pas reconnaître une incommensurabilité de principe à tout événement proprement dit et, par conséquent, détacher à nouveau la dimension herméneutique-narrative des déterminations quantitatives ou relations mathématiques ? On sait; d'ailleurs, à quel point la dimension herméneutique et même la notion de sens sont suspectées ou récusées par des auteurs qui pensent l'événement comme pluralité irréductibles ${ }^{20}$. "Un événement est toujours localisable ${ }^{21}$ : d'accord avec Badiou sur ce point, nous récusons comme lui toute notion de sens "pur" ou à plus forte raison toute postulation d'une réconciliation métaphysique (mème par le biais herméneutique) de l'histoire. Il n'en reste pas moins qu'un "site événementiel "se distingue d'une situation naturelle (qui, tout en étant déterminée, nous serait indifférente) : Badiou lui-mème retient l'historicité comme "critère local ${ }^{22}$ ". Cela veut dire que l'historicité ne peut pas ne pas être condition de l'événement, mais en fonction de déterminations précises. L' événement "quantique est incommensurable à l'événement historique et se situe à la limite extrème de toute événementialité : il n'en reste pas moins que ce qui permet de lui prēter cette qualité, c'est bien une " discontinuité révélatrice ", c'està-dire une rupture dans le champ du déterminisme absolu et une interrogation, fût-elle minimale. C'est ce qui autorise Deleuze, après

19. Alexandre Kojève, Introduction à la lecture de Hegeh Paris, Gallimard, 1947 , p. $369, n$. 1 .

20. Voir Gilles Deleuze, "Qu'est-ce qu'un événement? ", chap. 6 in Le pli, Paris, Éditions de Minuit, 1988 ; Alain Badiou, L'étre et l'événement, Paris, Éditions du Seuil, 1988, chap. IV et V.

21. Alain Badiou, op. cit., p. 199.

22. Ibid., p 197. 
Leibniz et Whitehead, à comprendre l'événement comme harmonique ou intensité, et à suggérer qu'il " $y$ a toujours quelque chose de psychique dans l'événement ${ }^{23}$ ".

La mesure n'est pas pour autant oubliée. L'incommensurabilité de l'événement est à concevoir, non comme un en soi ou une dimension autonome : plutôt comme une déchirure dans un tissu, la marque ou la remarque dans un texte, dotée du statut paradoxal d'une incommensurabilitè toujours relative, contextualisée, incarnée. L'événement a ainsi un caractère à la fois déterminè et surnuméraire, comme une occurrence musicale. En lui, les deux sens de la mesure communiquent : sa localisation quantitative le situe, mais ne l'épuise pas en tant qu'événement.

Nous sommes passé du temps de la nature au problème de l'événement par le fil conducteur de la mesure. Ce faisant, nous avons paru abandonner les problèmes du temps physique pour aborder l'incommensurabilité dans l'histoire. Mais, en fait, de même que l'incommensurabilité de l'événement historique se détache sur une chronologie, les mesures d'" événements " naturels ne prennent leur sens que rapportées à des contextes significatifs (en particulier, ceux du "discours " scientifique).

La fracture entre temps cosmologique et temps phénoménologique, déplorée par Ricoeur, n'est donc pas irrémédiable. Le temps calendaire constitue déjà un "tiers temps ", mais il faut saisir, plus généralement, l'interaction entre l'intérieur et l'extérieur, à tous les niveaux. La temporalité, révélation la plus subtile de la complexité, n'est ni purement naturelle ni seulement humaine. Elle déploie la dimension la plus révélatrice du dialogue entre l'homme et la nature, entre l'homme et ce qui le dépasse, dialogue et jeu constant entre nos mesures et leurs contreparties d'incommensurabilité.

Département de philosophie-Centre d'histoire des idées Université de Nice - Sophia Antipolis 